\title{
A retrospective study to reveal factors associated with postoperative shoulder imbalance in patients with adolescent idiopathic scoliosis with double thoracic curve
}

\author{
Choon Sung Lee, MD, PhD, Chang Ju Hwang, MD, PhD, Eic Ju Lim, MD, Dong-Ho Lee, MD, PhD, \\ and Jae Hwan Cho, MD
}

Department of Orthopaedic Surgery, Asan Medical Center, University of Ulsan College of Medicine, Seoul, South Korea

OBJECTIVE Postoperative shoulder imbalance (PSI) is a critical consideration after corrective surgery for a double thoracic curve (Lenke Type 2); however, the radiographic factors related to PSI remain unclear. The purpose of this study was to identify the radiographic factors related to PSI after corrective surgery for adolescent idiopathic scoliosis (AIS) in patients with a double thoracic curve.

METHODS This study included 80 patients with Lenke Type 2 AIS who underwent corrective surgery. Patients were grouped according to the presence [PSI(+)] or absence [PSI(-)] of shoulder imbalance at the final follow-up examination (differences of 20,15, and $10 \mathrm{~mm}$ were used). Various radiographic parameters, including the Cobb angle of the proximal and middle thoracic curves (PTC and MTC), radiographic shoulder height (RSH), clavicle angle, T-1 tilt, trunk shift, and proximal and distal wedge angles (PWA and DWA), were assessed before and after surgery and compared between groups.

RESULTS Overall, postoperative RSH decreased with time in the PSI(-) group but not in the PSI(+) group. Statistical analyses revealed that the preoperative Risser grade $(p=0.048)$, postoperative PWA $(p=0.028)$, and postoperative PTC/MTC ratio $(p=0.011)$ correlated with PSI. Presence of the adding-on phenomenon was also correlated with PSI, although this result was not statistically significant $(p=0.089)$.

CONCLUSIONS Postoperative shoulder imbalance is common after corrective surgery for Lenke Type 2 AIS and correlates with a higher Risser grade, a larger postoperative PWA, and a higher postoperative PTC/MTC ratio. Presence of the distal adding-on phenomenon is associated with an increased PSI trend, although this result was not statistically significant. However, preoperative factors other than the Risser grade that affect the development of PSI were not identified by the study. Additional studies are required to reveal the risk factors for the development of PSI.

http://thejns.org/doi/abs/10.3171/2016.6.PEDS16162

KEY WORDS adolescent idiopathic scoliosis; double thoracic curve; shoulder imbalance; proximal wedge angle; adding-on phenomenon; spine

\footnotetext{
ABBREVIATIONS AIS = adolescent idiopathic scoliosis; $C C A D=$ clavicle chest cage angle difference; $C S V L=$ central sacral vertical line; $D W A=$ distal wedge angle; LIV = lower instrumented vertebra; $\mathrm{MTC}=$ middle thoracic curve; $\mathrm{PSI}=$ postoperative shoulder imbalance; $\mathrm{PTC}=$ proximal thoracic curve; $\mathrm{PWA}=$ proximal wedge angle; $\mathrm{RSH}=$ radiographic shoulder height; UIV = upper instrumented vertebra.
}

SUBMITTED March 13, 2016. ACCEPTED June 30, 2016.

INCLUDE WHEN CITING Published online September 23, 2016; DOI: 10.3171/2016.6.PEDS16162. 
$\mathrm{C}$ ORRECTIVE surgery is considered the only definite treatment option for adolescent idiopathic scoliosis (AIS) in patients with a large curve $\left(>50^{\circ}\right)$. The standard for judging the success of corrective surgery depends on radiographic parameters and functional outcomes. Until now, although a scoring system for assessing functional outcomes in patients with deformity had been introduced, radiographic parameters have been frequently used to evaluate outcomes after surgery. In general, patients are satisfied if a balanced spine, correction of hump deformity, and balanced shoulders result from surgery. Self-esteem is also elevated with these positive morphological changes. Although a balanced spine or hump correction can be achieved through surgical treatment, including use of the rod derotation method or thoracoplasty, achieving shoulder balance is relatively unpredictable.

Although subjective shoulder balance is most important, it is hard to evaluate and achieve using conventional surgeries. Thus, radiographic shoulder balance is frequently evaluated instead of subjective shoulder balance and is also considered a critical consideration when judging the success of corrective surgery. Historically, recognizing the proximal thoracic curve (PTC) has been considered important, because coronal decompensation or postoperative shoulder imbalance (PSI) can result when the PTC is left unfused. ${ }^{7,8}$ Shoulder imbalance after surgery has been thought to be caused by the surgeon's failure to properly select the upper instrumented vertebra (UIV). Thus, previous studies examined the relationship between the upper level of instrumentation and PSI. ${ }^{3,5}$ However, controversial results in this regard have been reported in several previous studies. A few studies have also investigated other factors related to PSI, ${ }^{11,12}$ but no definite conclusions have been made.

Determining preoperative factors that predict PSI should be valuable in reducing the rate of PSI. In addition, correlation analysis between postoperative radiographic parameters and the occurrence of PSI might help enhance our understanding of this phenomenon. Thus, in this study, we aimed to identify the preoperative and postoperative radiographic parameters related to PSI after corrective surgery for AIS in patients with a double thoracic curve. The identification of these factors might help us identify possible causes of PSI and preventive methods.

\section{Methods}

This study included 80 patients who underwent corrective surgery for AIS with a double thoracic curve (Lenke Type 2) between January 2006 and December 2011 at our hospital. Patients with other types of idiopathic scoliosis and patients with less than 2 years of follow-up were excluded from the study. Demographic data, including sex, age, height, and weight, were obtained from the patients' electronic medical records. The radiographic data were obtained from whole-spine anteroposterior and lateral radiographs using the picture-archiving communication system at our institution. Plain radiographs were obtained with the patients in an upright standing position in both views and with their arms folded forward in the lateral views.

Each patient underwent posterior surgery using the rod derotation method with pedicle screw fixation. Selection of the fusion level largely depended on the patient's Lenke classification. Pedicle screw constructs were used at every level for each patient. However, forgoing screw insertion was considered for the level at which the width of the pedicle was too narrow. Osteotomies were not performed regardless of the rigidity of the curve. Patients received regular follow-up examinations 1, 6, and 12 months after surgery and yearly thereafter. This study was approved by the institutional review board of our institution, which waived the requirement for informed consent because of the retrospective nature of the analysis.

\section{Radiographic Assessment}

The degrees of PTC and middle thoracic curve (MTC) were assessed before and after surgery using the Cobb angle method. The PTC/MTC ratio was also calculated. Coronal-plane trunk shift was defined as the distance between the C-7 plumb line and the central sacral vertical line (CSVL). Coronal-plane decompensation was defined as a trunk shift $>20 \mathrm{~mm}$. Radiographic shoulder height (RSH) was defined as the difference between the horizontal lines passing both superolateral tips of the clavicles. The clavicle angle was defined as the angle between the line passing the highest points of both clavicles and the horizontal line. T-1 tilt was defined as the angle between the upper endplate of T-1 and the horizontal line. The clavicle chest cage angle difference was defined as the difference between the left and right clavicle chest cage angles. ${ }^{19}$ Risser grades ${ }^{16}$ were also measured preoperatively. The proximal wedge angle (PWA) and distal wedge angle (DWA) were assessed as the angle of coronal wedging in the disc space just proximal to the UIV or just distal to the lower instrumented vertebra (LIV), respectively. All radiographic measurements are summarized in Fig. 1.

Selective thoracic fusion was defined as a discontinuation of distal fusion at L-1 or higher. The adding-on phenomenon was defined as a progressive increase in the number of vertebrae included within the primary curve distally in combination with either an increase of $>5 \mathrm{~mm}$ in the deviation of the first vertebra below the instrumentation from the CSVL or an increase of $>5^{\circ}$ in angulation of the first disc below the instrumentation. ${ }^{18}$

We defined PSI as positive when the absolute value of RSH was $>20,15$, or $10 \mathrm{~mm}$. Patients were stratified according to the presence [PSI(+)] or absence [PSI(-)] of PSI at the final follow-up examination. All radiographic parameters were measured by one rater (J.H.C.) who at the time had 5 years of experience performing spinal surgery.

\section{Statistical Analysis}

Demographic data and radiographic parameters were compared between the PSI(+) and PSI(-) groups using the Student t-test, chi-square test, or Fisher exact test. Changes in the radiographic parameters between the preoperative and postoperative periods were analyzed using the paired t-test. Serial changes in RSH during the follow-up period were compared between groups using repeated-measures ANOVA. Comparisons of the radiographic parameters according to UIV were also performed using the Student t- 


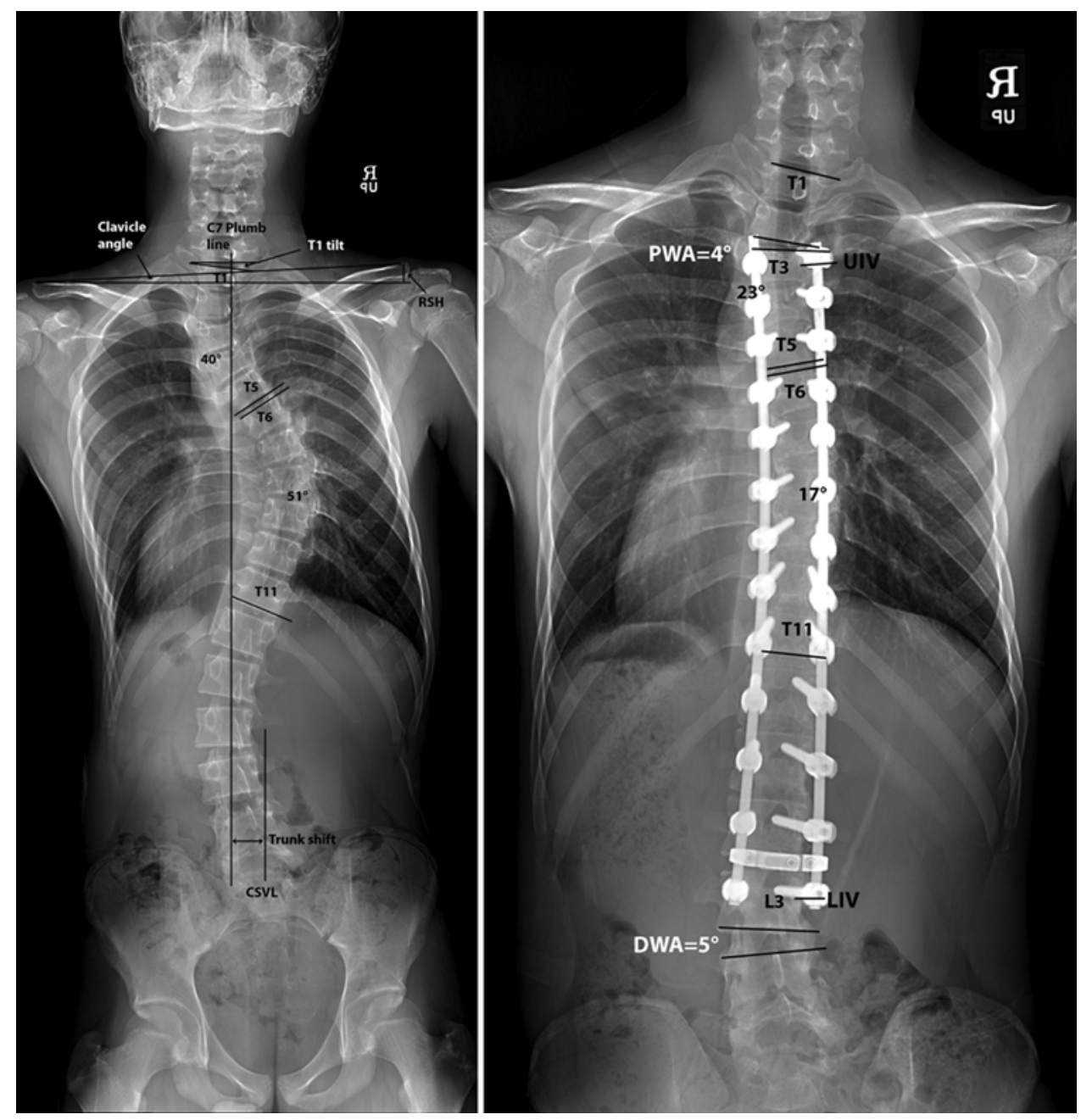

FIG. 1. Measurements of radiographic parameters. Left: Preoperative radiograph showing Lenke Type 2 AIS with a $40^{\circ} \mathrm{PTC}$ (T1T5) and $51^{\circ}$ MTC (T6-T11). Right: Postoperative radiograph showing the correction of PTC to $23^{\circ}$ and MTC to $17^{\circ}$.

test. The relationship between the adding-on phenomenon and PSI was assessed using the Fisher exact test. Multivariate logistic regression analysis was used to determine the radiographic factors related to PSI. As a sensitivity analysis, these analyses were performed repeatedly using the different criteria for PSI. Statistical analyses were performed using the Statistical Package for Social Sciences 21.0 software package (SPSS, Inc.); $p<0.05$ indicates a statistically significant result. Mean values are presented $\pm \mathrm{SD}$.

\section{Results}

\section{Demographic Data and Characteristics of Curves}

Of the 80 patients enrolled in this study, 26 were male and 54 were female. Their mean age was $15.1 \pm 2.7$ years. The mean height of the patients was $161.3 \pm 8.3 \mathrm{~cm}$, and their mean weight was $50.0 \pm 9.3 \mathrm{~kg}$. The mean followup period was $40.0 \pm 15.7$ months. The specific Lenke types included were 2A- (27 patients), 2AN (28 patients), 2B- (12 patients), 2BN (7 patients), 2C- (3 patients), and $2 \mathrm{CN}$ ( 3 patients). The mean Cobb angles of the PTC and MTC were $40.5^{\circ} \pm 9.9^{\circ}$ and $56.4^{\circ} \pm 10.7^{\circ}$, respectively.
The mean RSH, clavicle angle, and T-1 tilt were $-5.3 \pm$ $9.4 \mathrm{~mm},-0.3^{\circ} \pm 1.6^{\circ}$, and $5.8^{\circ} \pm 4.7^{\circ}$, respectively. T- 3 was the most frequently chosen UIV (47 patients), followed by T-4 (17 patients), T-2 (14 patients), T-1 (1 patient), and T-5 (1 patient). L-2 was the most frequently chosen LIV (34 cases), followed by L-1 (24 cases), L-3 (19 cases), T-12 (2 patients), and L-4 (1 patient).

\section{Comparisons of the PSI(+) and PSI(-) Groups}

The PSI was positive in $12(15 \%)$ of 80 patients at the final follow-up when using the criterion of $20 \mathrm{~mm}$. The proportions of patients with PSI increased to $22.5 \%$ (18 of 80 ) and $46.3 \%$ (37 of 80) when using the criteria of 15 and $10 \mathrm{~mm}$, respectively.

Demographic data and preoperative radiographic parameters were compared between the PSI(+) and PSI(-) groups using different criteria (Table 1). No differences were found between these groups except in the degree of preoperative MTC and Risser grade. A Risser grade higher than 3 was found more frequently in the PSI(+) group. The RSH increased postoperatively in both groups. However, the RSH decreased from $10.6 \mathrm{~mm} 1$ month after surgery 
TABLE 1. Comparisons of demographic data and preoperative radiographic parameters between the PSI(+) and PSI(-) groups according to different criteria*

\begin{tabular}{|c|c|c|c|c|c|c|c|c|c|}
\hline $\begin{array}{c}\text { Demographic or } \\
\text { Radiographic Parameter }\end{array}$ & $\begin{array}{c}\text { SHD }<20 \mathrm{~mm} \\
\quad(n=68)\end{array}$ & $\begin{aligned} & \mathrm{SHD} \geq 20 \mathrm{~mm} \\
&(\mathrm{n}=12)\end{aligned}$ & $\begin{array}{c}p \\
\text { Value }\end{array}$ & $\begin{array}{c}\text { SHD }<15 \mathrm{~mm} \\
\quad(\mathrm{n}=62)\end{array}$ & $\begin{aligned} \text { SHD } & \geq 15 \mathrm{~mm} \\
(\mathrm{n} & =18)\end{aligned}$ & $\begin{array}{c}\mathrm{p} \\
\text { Value }\end{array}$ & $\begin{array}{c}\text { SHD }<10 \mathrm{~mm} \\
\quad(\mathrm{n}=43)\end{array}$ & $\begin{aligned} \text { SHD } & \geq 10 \mathrm{~mm} \\
(\mathrm{n} & =37)\end{aligned}$ & $\begin{array}{c}\mathrm{p} \\
\text { Value }\end{array}$ \\
\hline Age (yrs) & $15.1 \pm 2.8$ & $14.9 \pm 1.8$ & 0.799 & $15.1 \pm 2.8$ & $15.1 \pm 2.1$ & 0.937 & $15.3 \pm 3.0$ & $14.9 \pm 2.2$ & 0.513 \\
\hline $\operatorname{Sex}(M / F)$ & $22: 46$ & $4: 8$ & 0.947 & $20: 42$ & $6: 12$ & 0.932 & $14: 29$ & $12: 25$ & 0.990 \\
\hline Height (cm) & $161.5 \pm 8.6$ & $160.2 \pm 6.4$ & 0.635 & $161.4 \pm 8.7$ & $160.9 \pm 6.9$ & 0.818 & $162.3 \pm 8.9$ & $160.1 \pm 7.6$ & 0.232 \\
\hline Weight (kg) & $49.5 \pm 9.2$ & $53.5 \pm 9.7$ & 0.215 & $49.5 \pm 9.2$ & $51.9 \pm 10.0$ & 0.345 & $49.5 \pm 9.9$ & $50.7 \pm 8.7$ & 0.571 \\
\hline $\operatorname{PTC}\left({ }^{\circ}\right)$ & $41.1 \pm 10.3$ & $37.2 \pm 6.6$ & 0.201 & $41.3 \pm 10.4$ & $38.0 \pm 7.7$ & 0.216 & $42.0 \pm 11.3$ & $38.8 \pm 7.7$ & 0.141 \\
\hline $\operatorname{MTC}\left({ }^{\circ}\right)$ & $57.5 \pm 10.8$ & $49.8 \pm 7.3$ & $0.021 \dagger$ & $57.0 \pm 10.4$ & $54.2 \pm 11.8$ & 0.338 & $56.5 \pm 10.3$ & $56.2 \pm 11.3$ & 0.894 \\
\hline PTC/MTC ratio & $0.72 \pm 0.13$ & $0.75 \pm 0.10$ & 0.456 & $0.73 \pm 0.13$ & $0.71 \pm 0.12$ & 0.718 & $0.74 \pm 0.14$ & $0.70 \pm 0.11$ & 0.156 \\
\hline $\mathrm{RSH}(\mathrm{mm})$ & $-5.4 \pm 9.7$ & $-4.5 \pm 8.1$ & 0.748 & $-5.4 \pm 9.6$ & $-4.9 \pm 9.2$ & 0.831 & $-5.5 \pm 9.8$ & $-5.0 \pm 9.1$ & 0.818 \\
\hline Clavicle angle $\left({ }^{\circ}\right)$ & $-0.3 \pm 1.8$ & $-0.3 \pm 1.4$ & 0.936 & $-0.3 \pm 1.8$ & $-0.3 \pm 1.7$ & 0.979 & $-0.3 \pm 1.9$ & $-0.2 \pm 1.6$ & 0.738 \\
\hline T-1 tilt $\left(^{\circ}\right)$ & $5.7 \pm 4.7$ & $5.9 \pm 4.6$ & 0.893 & $6.0 \pm 4.6$ & $5.1 \pm 5.0$ & 0.477 & $6.3 \pm 4.6$ & $5.1 \pm 4.7$ & 0.219 \\
\hline Risser grade $(\leq 2 / \geq 3)$ & $26 / 42$ & $1 / 11$ & 0.051 & $25 / 37$ & $2 / 16$ & $0.024 \dagger$ & $16 / 27$ & $11 / 26$ & 0.481 \\
\hline $\operatorname{CCAD}\left({ }^{\circ}\right)$ & $11.5 \pm 9.5$ & $10.8 \pm 7.6$ & 0.805 & $11.0 \pm 8.9$ & $12.7 \pm 10.6$ & 0.499 & $9.7 \pm 9.3$ & $13.4 \pm 8.9$ & 0.073 \\
\hline Trunk shift (mm) & $2.6 \pm 12.9$ & $-2.5 \pm 12.9$ & 0.230 & $2.7 \pm 12.9$ & $-1.1 \pm 13.1$ & 0.287 & $1.7 \pm 12.3$ & $1.9 \pm 13.9$ & 0.943 \\
\hline FU period (mos) & $29.8 \pm 15.7$ & $31.1 \pm 16.2$ & 0.788 & $29.5 \pm 15.8$ & $31.5 \pm 15.7$ & 0.639 & $29.3 \pm 17.0$ & $30.7 \pm 14.2$ & 0.704 \\
\hline
\end{tabular}

$\mathrm{FU}=$ follow-up; $\mathrm{SHD}=$ shoulder height difference.

* Data represent mean \pm SD for continuous variables and number of patients for categorical variables.

$\dagger$ Statistically significant value.

to $6.7 \mathrm{~mm}$ at the final follow-up examination in the PSI(-) group $(\mathrm{p}=0.001)$. This trend was not evident in the PSI $(+)$ group (which changed from 23.0 to $24.0 \mathrm{~mm}$ in the same period; $\mathrm{p}=0.735)$. The changes in RSH during the postoperative period (summarized in Table 2) were significantly different between the PSI(+) and PSI(-) groups according to repeated-measures ANOVA $(\mathrm{p}<0.001)$.

One month after surgery, the PWA was larger in the PSI(+) group than in the PSI $(-)$ group $\left(6.4^{\circ} \pm 4.9^{\circ}\right.$ vs $3.2^{\circ}$ $\pm 3.3^{\circ}$, respectively; $\left.\mathrm{p}=0.005\right)$. However, no difference was found in terms of DWA $\left(1.7^{\circ} \pm 2.5^{\circ}\right.$ vs $2.4^{\circ} \pm 2.4^{\circ}$, respectively; $p=0.354)$. At the final follow-up, the same results were found for PWA and DWA. In addition, the radiographic parameters related to shoulder balance, such as $\mathrm{RSH}$, clavicle angle, and T-1 tilt, were significantly different between the 2 groups. Furthermore, the PTC/MTC ratio was higher in the PSI(+) group than in the PSI(-) group (2.15 vs 1.59 , respectively; $\mathrm{p}=0.022$ ). These differences decreased when the criterion was changed from 20 to 15 or $10 \mathrm{~mm}$. Comparisons of the radiographic parameters at the final follow-up between these 2 groups according to these criteria are summarized in Table 3.

\section{Effects of the Adding-On Phenomenon and UIV on PSI}

The distal adding-on phenomenon was observed in 6 patients during the follow-up period. The relationships between the distal adding-on phenomenon and PSI are compared in Table 4. There were positive correlations between these 2 factors using the criteria of 20 and $15 \mathrm{~mm}$ ( $\mathrm{p}=$ 0.041 and 0.021 , respectively). The LIVs for the patients with the adding-on phenomenon were L-1 in 3 patients, L-2 in 1 patient, and L-3 in 2 patients. No correlation was found between LIV and the adding-on phenomenon ( $\mathrm{p}=$ 0.405 ). Preoperative and postoperative radiographic parameters are compared in Table 5 according to the UIV.
A large preoperative PTC and large T-1 tilt were related to selecting a higher fusion level (UIV $=\mathrm{T}-1$ or $\mathrm{T}-2)$. Of the postoperative radiographic parameters, RSH and PWA were related to UIV. Radiographic shoulder height (4.3 vs $10.5 \mathrm{~mm}$, respectively) and PWA ( $1.1^{\circ}$ vs $4.2^{\circ}$, respectively) were lower in the group of patients with a higher fusion level (UIV = T-1 or T-2) than in the group of those with a lower fusion level.

\section{Multivariate Analysis}

A multivariate logistic regression analysis was conducted using 6 variables: Risser grade at the time of surgery, 3 postoperative radiographic parameters (PWA, T-1 tilt, and PTC/MTC ratio), UIV, and presence of the adding-on phenomenon. Of these 6 variables, Risser grade, postoperative PWA, and the PTC/MTC ratio were found to be related to PSI ( $\mathrm{p}=0.048,0.028$, and 0.011 , respectively). Presence of the adding-on phenomenon also was found to be related to PSI, but it was not statistically significant ( $\mathrm{p}=$

TABLE 2. Changes in RSH during the postoperative follow-up periods in both groups*

\begin{tabular}{lccr}
\hline \multicolumn{1}{c}{ FU Period } & PSI $(-)(n=68)$ & $P S I(+)(n=12)$ & $p$ Value \\
\hline Preop $\dagger$ & $-5.4 \pm 9.7$ & $-4.5 \pm 8.1$ & 0.748 \\
\hline 1-mo postop & $10.6 \pm 9.4$ & $23.0 \pm 9.4$ & $<0.001$ \\
\hline 6-mo postop & $10.6 \pm 10.1$ & $17.1 \pm 9.9$ & 0.062 \\
\hline 1-yr postop & $8.7 \pm 8.6$ & $19.4 \pm 10.2$ & 0.001 \\
\hline Final FU & $6.7 \pm 7.1$ & $24.0 \pm 2.3$ & $<0.001$ \\
\hline
\end{tabular}

* PSI was defined by the criteria of $20 \mathrm{~mm}$. Data represent mean \pm SD. PSI values represent millimeters.

† Negative value indicates that the right shoulder was more elevated than the left shoulder. 
TABLE 3. Comparisons of radiographic parameters at final follow-up between the PSI(+) and PSI(-) groups according to different criteria*

\begin{tabular}{|c|c|c|c|c|c|c|c|c|c|}
\hline Radiographic Parameter & $\begin{array}{c}\text { SHD }<20 \mathrm{~mm} \\
\quad(n=68)\end{array}$ & $\begin{aligned} & \mathrm{SHD} \geq 20 \mathrm{~mm} \\
&(\mathrm{n}=12)\end{aligned}$ & $\begin{array}{c}p \\
\text { Value }\end{array}$ & $\begin{array}{c}\text { SHD }<15 \mathrm{~mm} \\
(\mathrm{n}=62)\end{array}$ & $\begin{aligned} & \text { SHD } \geq 15 \mathrm{~mm} \\
&(\mathrm{n}=18)\end{aligned}$ & $\begin{array}{c}p \\
\text { Value }\end{array}$ & $\begin{array}{c}\mathrm{SHD}<10 \mathrm{~mm} \\
(\mathrm{n}=43)\end{array}$ & $\begin{aligned} \text { SHD } & \geq 10 \mathrm{~mm} \\
(\mathrm{n} & =37)\end{aligned}$ & $\begin{array}{c}p \\
\text { Value }\end{array}$ \\
\hline PTC $\left({ }^{\circ}\right)$ & $20.0 \pm 7.2$ & $21.4 \pm 8.9$ & 0.558 & $19.8 \pm 7.2$ & $21.7 \pm 8.1$ & 0.342 & $20.8 \pm 7.8$ & $19.6 \pm 7.0$ & 0.505 \\
\hline Correction rate of PTC (\%) & $50.6 \pm 15.1$ & $43.4 \pm 16.1$ & 0.137 & $51.3 \pm 15.5$ & $43.6 \pm 13.5$ & 0.061 & $49.9 \pm 15.9$ & $49.1 \pm 14.9$ & 0.804 \\
\hline $\operatorname{MTC}\left({ }^{\circ}\right)$ & $13.7 \pm 5.4$ & $11.2 \pm 5.0$ & 0.134 & $13.5 \pm 5.4$ & $12.6 \pm 5.6$ & 0.495 & $14.3 \pm 5.6$ & $12.2 \pm 5.0$ & 0.096 \\
\hline Correction rate of MTC $(\%)$ & $75.9 \pm 8.8$ & $78.1 \pm 7.4$ & 0.420 & $76.0 \pm 8.9$ & $77.1 \pm 7.8$ & 0.624 & $74.5 \pm 9.3$ & $78.2 \pm 7.4$ & 0.051 \\
\hline PTC/MTC ratio & $1.59 \pm 0.66$ & $2.15 \pm 1.22$ & $0.022 \ddagger$ & $1.59 \pm 0.67$ & $1.97 \pm 1.07$ & 0.067 & $1.56 \pm 0.66$ & $1.81 \pm 0.90$ & 0.154 \\
\hline $\mathrm{RSH}(\mathrm{mm})$ & $6.7 \pm 7.1$ & $24.0 \pm 2.3$ & $<0.001 \ddagger$ & $5.7 \pm 6.7$ & $21.6 \pm 4.0$ & $<0.001 \ddagger$ & $3.6 \pm 4.8$ & $15.9 \pm 8.4$ & $<0.001 \neq$ \\
\hline Clavicle angle $\left({ }^{\circ}\right)$ & $1.5 \pm 1.4$ & $6.5 \pm 6.7$ & $<0.001 \ddagger$ & $1.3 \pm 1.3$ & $5.3 \pm 5.7$ & $0.009 \ddagger$ & $0.9 \pm 1.1$ & $3.8 \pm 4.3$ & $<0.001 \ddagger$ \\
\hline T-1 tilt $\left({ }^{\circ}\right)$ & $8.1 \pm 4.3$ & $11.6 \pm 6.4$ & $0.019 \ddagger$ & $8.2 \pm 4.2$ & $10.2 \pm 6.2$ & 0.112 & $8.4 \pm 4.4$ & $8.9 \pm 5.5$ & 0.676 \\
\hline STF (yes/no) & $22 / 46$ & $4 / 8$ & 0.947 & $20 / 42$ & $6 / 12$ & 0.932 & $17 / 26$ & $9 / 28$ & 0.148 \\
\hline Trunk shift (mm)† & $-7.5 \pm 8.9$ & $-5.4 \pm 8.8$ & 0.463 & $-7.2 \pm 8.9$ & $-7.0 \pm 9.1$ & 0.934 & $-8.0 \pm 9.1$ & $-6.3 \pm 8.7$ & 0.385 \\
\hline $\operatorname{PWA}\left({ }^{\circ}\right)$ & $2.4 \pm 2.8$ & $5.2 \pm 6.0$ & $0.013 \ddagger$ & $2.4 \pm 2.8$ & $4.5 \pm 5.0$ & 0.100 & $2.5 \pm 3.0$ & $3.3 \pm 4.0$ & 0.275 \\
\hline DWA $\left({ }^{\circ}\right)$ & $2.4 \pm 2.8$ & $1.8 \pm 2.2$ & 0.523 & $2.5 \pm 2.9$ & $1.7 \pm 1.9$ & 0.310 & $2.5 \pm 2.9$ & $2.1 \pm 2.6$ & 0.458 \\
\hline
\end{tabular}

STF = selective thoracic fusion.

* Data represent mean \pm SD.

$\uparrow$ Negative value indicates that the trunk was shifted to the left side.

$\ddagger$ Statistically significant value.

0.089). Representative radiographs of patients showing the factors related to PSI are shown in Figs. 2-5.

\section{Discussion}

Shoulder imbalance after corrective surgery for AIS is regarded as a critical problem, especially in patients with a double thoracic curve. In most instances, the height of the right shoulder becomes lower than that of the left shoulder after surgery. Thus, if the left shoulder is elevated preoperatively, extra caution is required to prevent PSI. ${ }^{17}$ However, the benefits of PTC correction are debatable. In an earlier study, spontaneous correction of PTCs after MTC correction was reported. ${ }^{6}$ In another study, it was suggested that extending fusion to T-2 or T-3 was not needed for nonstructural PTCs. ${ }^{3}$ However, the commonly recommended upper fusion level is T-2 for patients with AIS and a double thoracic curve. We did not extend proximal fusion to T-2 in many patients because it could not cause shoulder imbalance if the right shoulder was elevated preoperatively. We believed that stopping the fusion at T-3 would be enough if the PTC was not severe.

We aimed to serially demonstrate postoperative changes in shoulder balance and identify plausible radiographic factors related to PSI. In this analysis, we found that the difference in shoulder height decreased during the postoperative follow-up period. However, this spontaneous correction was not found in our PSI(+) group. In this group, shoulder height imbalance was maintained from the immediate postoperative period through to the final followup examination. Our data also suggest that the postoperative PWA, postoperative PTC/MTC ratio, and presence of the adding-on phenomenon are most likely to be factors related to PSI. In the PSI $(+)$ group, the PWA increased postoperatively, which can aggravate T-1 tilt and shoulder imbalance. The increase in the PWA is thought to be caused by a compensation mechanism aimed at maintaining trunk balance in the coronal plane. In this study, the PWA was also smaller when instrumentation was extended more proximally (Table 5), which can lead to trunk imbalance. However, we did not identify any positive relationship between trunk shift and UIV.

The postoperative PTC/MTC ratio might also be an important factor for the onset of PSI. Several studies have reported that PTC and MTC correction prevent PSI. Sufficient PTC correction that is matched with MTC correction has also been suggested to reduce PSI. ${ }^{13}$ It was also reported that shoulder balance did not sufficiently improve even after applying the direct vertebral rotation method, which means that less MTC correction is needed to achieve better shoulder balance. ${ }^{2}$ The results of our study are consistent with the findings of these previous studies.

The Risser grade is an indicator of maturity and is also associated with curve flexibility. If the Risser grade is higher, the ability to compensate for a shoulder imbalance might be reduced, which may underpin the observed correlation between the Risser grade and PSI.

The adding-on phenomenon is a frequently noted complication after inadequate LIV selection. ${ }^{10}$ Two studies addressed the relationship between this phenomenon and

TABLE 4. Comparisons of distal adding-on phenomenon between the $\mathrm{PSI}(+)$ and $\mathrm{PSI}(-)$ groups according to different criteria

\begin{tabular}{ccccc}
\hline & \multicolumn{3}{c}{ Adding-On Phenomenon } & \\
\cline { 2 - 4 } SHD $(\mathrm{mm})$ & Not Seen & Seen & Total & p Value \\
\hline$\geq 20$ & 65 & 3 & 68 & \\
\hline$<20$ & 9 & 3 & 12 & $0.041^{*}$ \\
\hline$\geq 15$ & 60 & 2 & 62 & \multirow{2}{*}{$0.021^{*}$} \\
\hline$<15$ & 14 & 4 & 18 & \\
\hline$\geq 10$ & 42 & 1 & 43 & \multirow{2}{*}{0.090} \\
\hline$<10$ & 32 & 5 & 37 & \\
\hline
\end{tabular}

* Statistically significant value. 
TABLE 5. Comparisons of preoperative and postoperative radiographic parameters according to the upper instrumented vertebra*

\begin{tabular}{|c|c|c|c|c|c|c|}
\hline \multirow[b]{2}{*}{ Radiographic Parameter } & \multicolumn{2}{|c|}{ UIV Before Surgery } & \multirow[b]{2}{*}{ p Value } & \multicolumn{2}{|c|}{ UIV After Surgery } & \multirow[b]{2}{*}{$\mathrm{p}$ Value } \\
\hline & $T 1-2(n=15)$ & T3-5 $(n=65)$ & & $T 1-2(n=15)$ & T3-5 $(n=65)$ & \\
\hline $\operatorname{PTC}\left({ }^{\circ}\right)$ & $46.7 \pm 13.6$ & $39.1 \pm 8.3$ & 0.006 & $18.5 \pm 5.4$ & $20.7 \pm 7.8$ & 0.305 \\
\hline $\operatorname{MTC}\left({ }^{\circ}\right)$ & $58.1 \pm 10.9$ & $56.0 \pm 10.7$ & 0.480 & $13.0 \pm 5.1$ & $13.4 \pm 5.5$ & 0.798 \\
\hline $\mathrm{RSH}(\mathrm{mm}) \dagger$ & $-3.4 \pm 11.0$ & $-5.7 \pm 9.1$ & 0.378 & $4.3 \pm 8.4$ & $10.5 \pm 8.9$ & 0.017 \\
\hline Clavicle angle $\left({ }^{\circ}\right) \ddagger$ & $-0.4 \pm 2.3$ & $-0.3 \pm 1.6$ & 0.785 & $1.3 \pm 1.6$ & $2.4 \pm 3.6$ & 0.255 \\
\hline T-1 tilt $\left({ }^{\circ}\right)$ & $8.1 \pm 5.2$ & $5.2 \pm 4.4$ & 0.032 & $8.1 \pm 3.5$ & $8.8 \pm 5.0$ & 0.644 \\
\hline Trunk shift $(\mathrm{mm}) \S$ & $-0.9 \pm 10.5$ & $2.5 \pm 13.5$ & 0.369 & $-9.0 \pm 9.4$ & $-6.8 \pm 8.8$ & 0.385 \\
\hline $\operatorname{PWA}\left({ }^{\circ}\right)$ & - & - & - & $1.1 \pm 1.6$ & $4.2 \pm 3.9$ & 0.005 \\
\hline DWA $\left({ }^{\circ}\right) \ddagger$ & - & - & - & $1.9 \pm 2.4$ & $2.4 \pm 2.5$ & 0.477 \\
\hline
\end{tabular}

\footnotetext{
* Data represent mean \pm SD.

$\dagger$ Negative value indicates that the right shoulder is more elevated than the left shoulder.

$\ddagger$ Negative value indicates that the right clavicle is more elevated than the left clavicle.

$\S$ Negative value indicates that the trunk is shifted to the left.
}

PSI. In one of these study reports, the clavicle angle was reported to be smaller in patients with the distal adding-on phenomenon, which means that the adding-on phenomenon could be a compensation mechanism for PSI. ${ }^{11}$ However, this result did not reach statistical significance $(\mathrm{p}=$ 0.09 ) and was confined to those with a Lenke 1A curve. It was reported from the other study that the adding-on phenomenon was significantly less common in the shoulderimbalance group of patients with a Lenke Type 2 curve. ${ }^{1}$ The authors suggested that both PSI and the adding-on phenomenon need to be prevented. However, on the basis of our results, this recommendation should be reevaluated, although the number of patients with the adding-on phenomenon was too small to suggest that this phenomenon was not a compensation mechanism for PSI.

T-1 tilt has been thought to be another factor that is possibly related to PSI. Although most patients with a double thoracic curve had a positive T-1 tilt, it was not correlated with PSI in our series, which is consistent with the results of other studies. ${ }^{7,9}$ However, additional studies are required
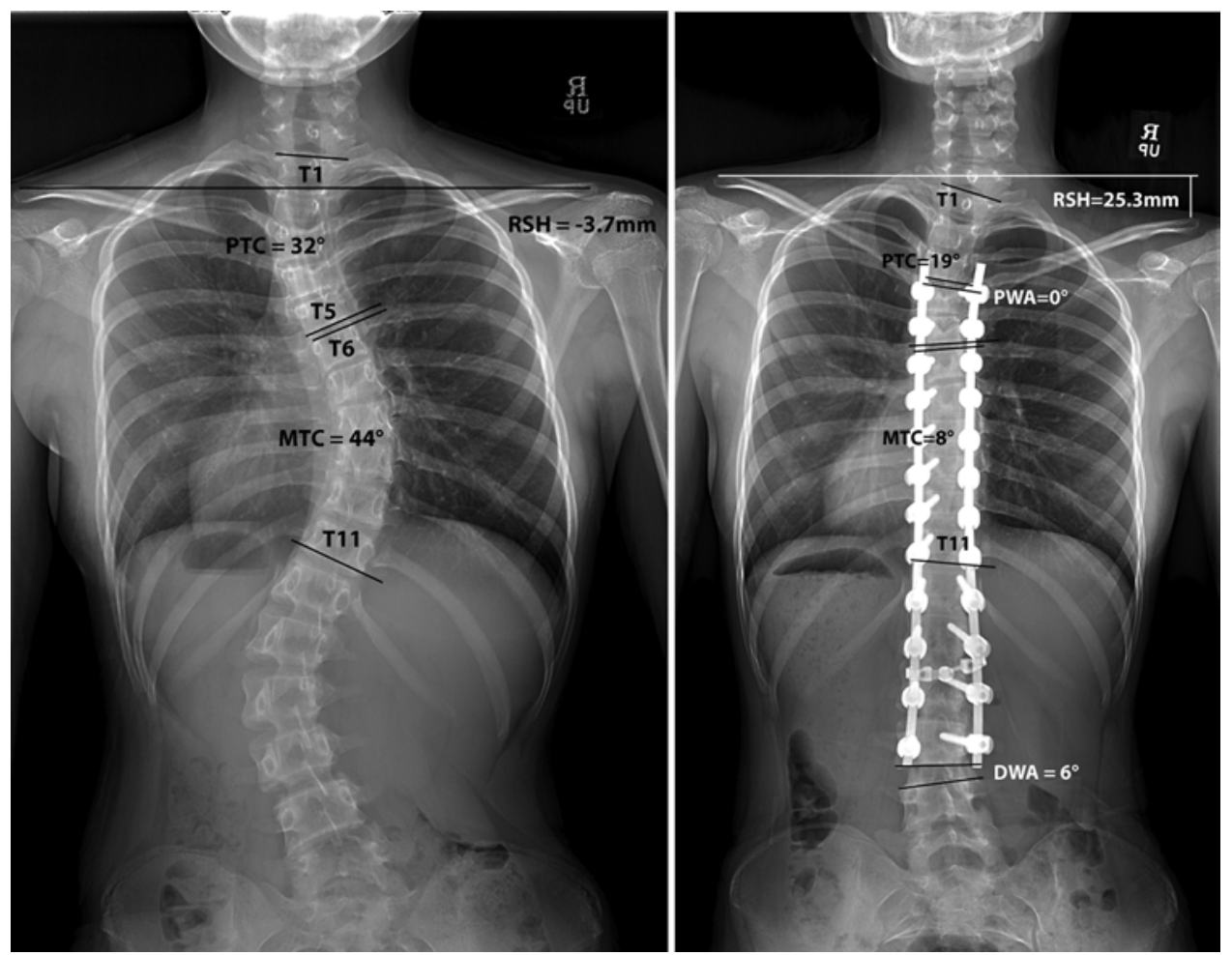

FIG. 2. Left: Preoperative radiograph of a 13-year-old girl showing well-balanced shoulders with a $32^{\circ} \mathrm{PTC}$ and $44^{\circ}$ MTC. Right: Postoperative radiograph 4 years after surgery showing shoulder imbalance (RSH $25.3 \mathrm{~mm}$ ), a $6^{\circ} \mathrm{DWA}$, and a high postoperative PTC/MTC ratio (2.4). 


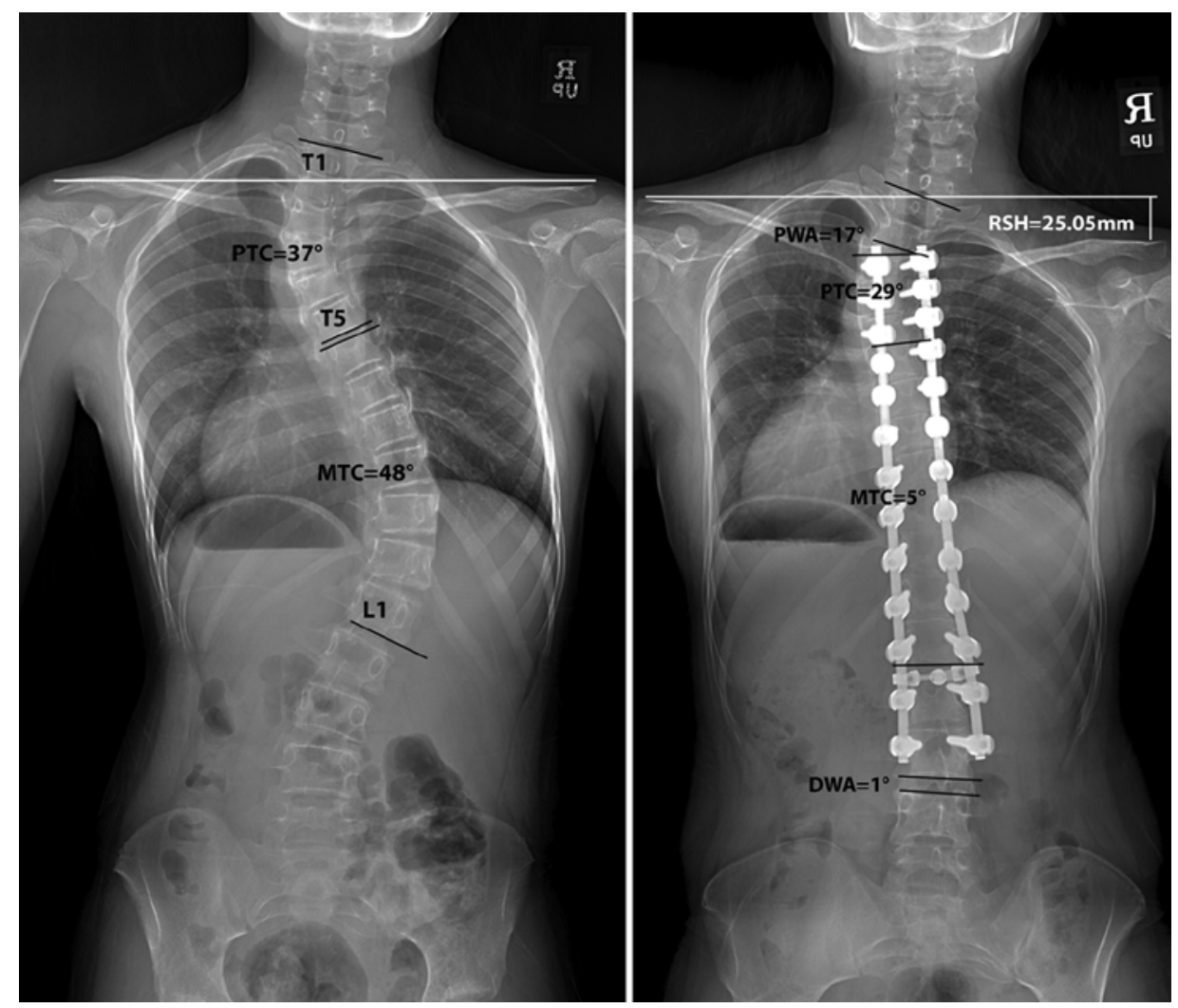

FIG. 3. Left: Preoperative radiograph of a 12-year-old girl showing well-balanced shoulders with a $37^{\circ} \mathrm{PTC}$ and $48^{\circ}$ MTC. Right: Postoperative radiograph 5 years after surgery showing shoulder imbalance (RSH $25.05 \mathrm{~mm}$ ), a $17^{\circ} \mathrm{PWA}$, and a high postoperative PTC/MTC ratio (5.8).

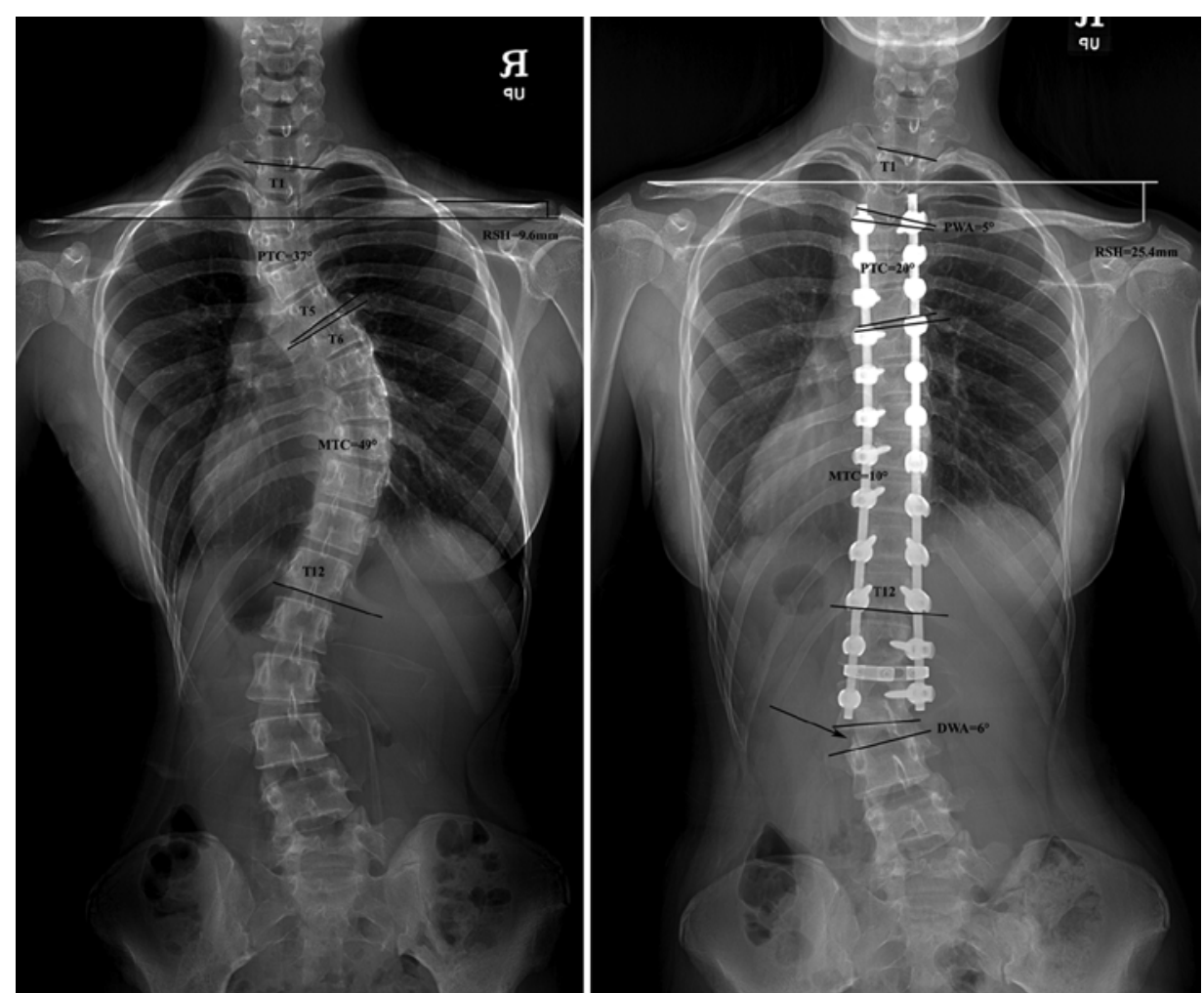

FIG. 4. Left: Preoperative radiograph of a 15-year-old girl showing well-balanced shoulders with a $37^{\circ}$ PTC and $49^{\circ}$ MTC. Right: Postoperative radiograph 2 years after surgery revealing shoulder imbalance (RSH $25.4 \mathrm{~mm}$ ) and a distal adding-on phenomenon (arrow). 

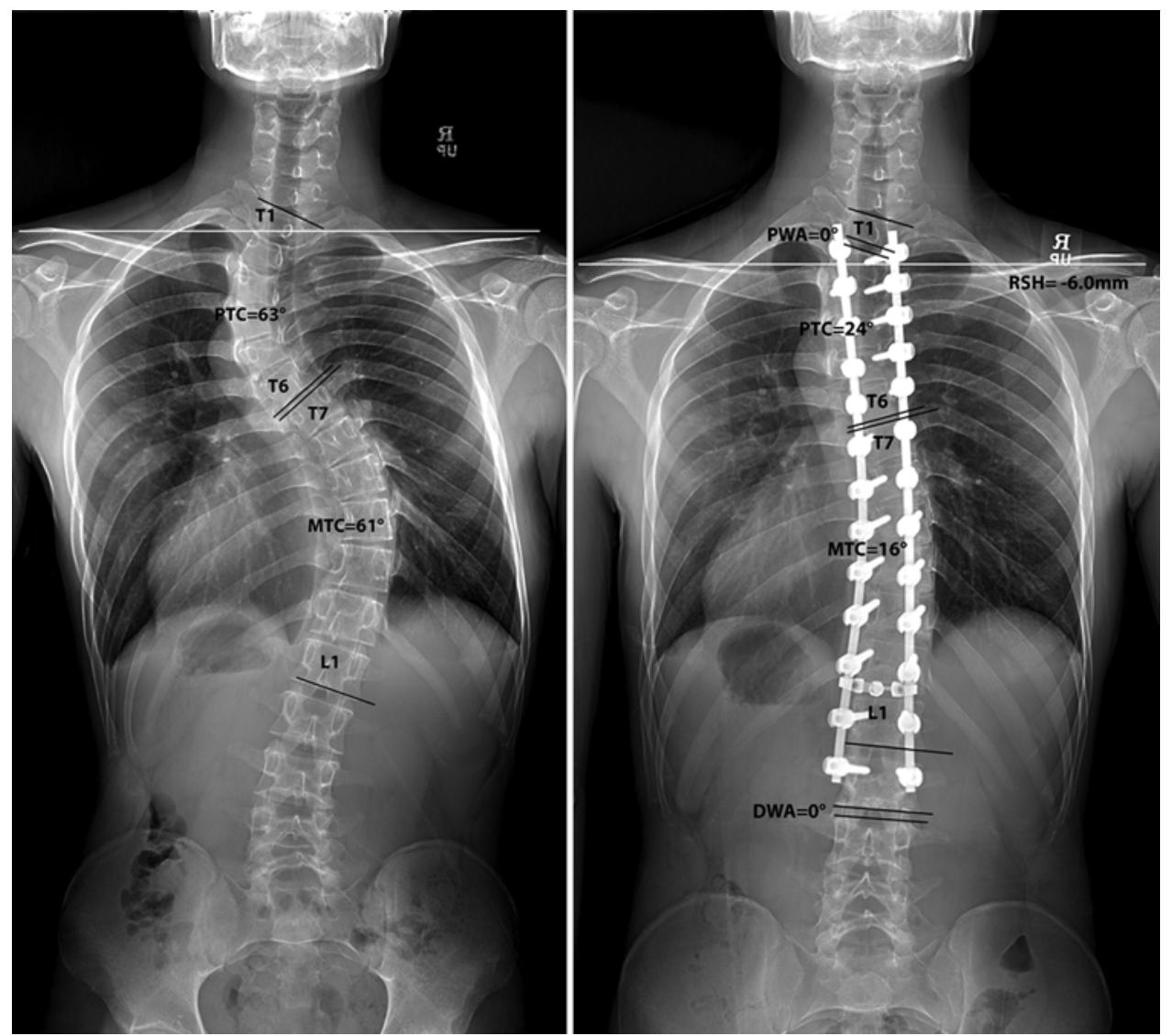

FIG. 5. Left: Preoperative radiograph of a 17-year-old boy showing well-balanced shoulders with a $63^{\circ} \mathrm{PTC}$ and $61^{\circ}$ MTC. Right: Postoperative radiograph 3 years after surgery showing balanced shoulders (RSH $-6.0 \mathrm{~mm})$, a $0^{\circ} \mathrm{PWA}$, a $0^{\circ} \mathrm{DWA}$, and a low postoperative PTC/MTC ratio (1.5).

in this regard, because in another study, ${ }^{5} \mathrm{~T}-1$ tilt was considered to be an important factor for selecting the proximal fusion level.

A preoperative clavicle chest cage angle difference (CCAD) was suggested recently as a new parameter for predicting PSI. ${ }^{19}$ However, we did not find any relationship between the preoperative CCAD and PSI in our study. Therefore, further validation of this issue is required.

There are several critical considerations that need to be addressed in relation to PSI. First, RSH is not a clinical indicator of shoulder imbalance. Moreover, clinical shoulder imbalance does not correlate with radiographic shoulder imbalance. Accordingly, the concept of "cosmetic shoulder balance" has been suggested..$^{15}$ Second, it is not clear how shoulder imbalance can be measured accurately, but several studies have reported the effectiveness of various methods. ${ }^{4,14,20}$ The clavicular angle and RSH are considered more reliable parameters according to a previous report. ${ }^{4}$ Third, the definition of PSI has not been clearly established. In the case of RSH, 10 and $20 \mathrm{~mm}$ were used as cutoff values in a number of studies. ${ }^{4,13}$ We used variable cutoff values of 20,15 , and $10 \mathrm{~mm}$ for sensitivity analysis. Fourth, the proximal fusion level for double thoracic idiopathic scoliosis has also been the subject of debate. Because of these complex considerations, interpreting our results is not completely straightforward.

This study had several notable limitations. First, our results failed to reveal a meaningful association between preoperative parameters and PSI. Thus, we could not determine which radiographic parameters are predictive of PSI. Only associations between PSI and postoperative factors were evident in our findings. Thus, our results do not suggest any methods that could prevent PSI. Second, the number of patients in our PSI(+) group who met the criterion of a $20-\mathrm{mm} \mathrm{RSH}$ was relatively small, and therefore, a study with a larger sample size is needed to validate our results. Third, the average follow-up period (40 months) in our patient cohort was somewhat insufficient to determine the presence of the adding-on phenomenon or decompensation. A longer follow-up duration is needed to reveal more clearly the relationship between PSI and these factors. Fourth, a lack of clinical analysis, such as analysis using the Scoliosis Research Society-22 (SRS-22) score, was also a limitation in that radiographic shoulder imbalance is different from clinical shoulder imbalance.

The above-mentioned limitations notwithstanding, however, we reveal here for the first time that PWA correlates with PSI, and our data support and refute previously suggested findings. We believe that this correlation, which was derived from both sensitivity and multivariate logistic regression analyses, is relatively reliable. Thus, the results of our study provide a meaningful basis for further evaluation of the relationship between PTC correction and PSI in patients with AIS and a double thoracic curve. 


\section{Conclusions}

PSI is correlated with a higher Risser grade, a larger postoperative PWA, and a higher postoperative PTC/MTC ratio in patients with AIS and a double thoracic curve. In addition, the presence of a distal adding-on phenomenon shows a correlation trend with an increasing PSI, although it was not found in our analysis to be statistically significant. However, no preoperative factor except for Risser grade affected the development of PSI. On the basis of these findings, more specific strategies might be required to prevent proximal wedging, unbalanced PTC/MTC correction, and the adding-on phenomenon. However, additional studies are required to reveal risk factors for the development of PSI.

\section{References}

1. Cao K, Watanabe K, Hosogane N, Toyama Y, Yonezawa I, Machida M, et al: Association of postoperative shoulder balance with adding-on in Lenke Type II adolescent idiopathic scoliosis. Spine (Phila Pa 1976) 39:E705-E712, 2014

2. Chang DG, Kim JH, Kim SS, Lim DJ, Ha KY, Suk SI: How to improve shoulder balance in the surgical correction of double thoracic adolescent idiopathic scoliosis. Spine (Phila Pa 1976) 39:E1359-E1367, 2014

3. Cil A, Pekmezci M, Yazici M, Alanay A, Acaroglu RE, Deviren V, et al: The validity of Lenke criteria for defining structural proximal thoracic curves in patients with adolescent idiopathic scoliosis. Spine (Phila Pa 1976) 30:2550-2555, 2005

4. Hong JY, Suh SW, Yang JH, Park SY, Han JH: Reliability analysis of shoulder balance measures: comparison of the 4 available methods. Spine (Phila Pa 1976) 38:E1684-E1690, 2013

5. Ilharreborde B, Even J, Lefevre Y, Fitoussi F, Presedo A, Souchet P, et al: How to determine the upper level of instrumentation in Lenke types 1 and 2 adolescent idiopathic scoliosis: a prospective study of 132 patients. J Pediatr Orthop 28:733-739, 2008

6. Kuklo TR, Lenke LG, Won DS, Graham EJ, Sweet FA, Betz RR, et al: Spontaneous proximal thoracic curve correction after isolated fusion of the main thoracic curve in adolescent idiopathic scoliosis. Spine (Phila Pa 1976) 26:1966-1975, 2001

7. Lee CK, Denis F, Winter RB, Lonstein JE: Analysis of the upper thoracic curve in surgically treated idiopathic scoliosis. A new concept of the double thoracic curve pattern. Spine (Phila Pa 1976) 18:1599-1608, 1993

8. Lenke LG, Bridwell KH, O'Brien MF, Baldus C, Blanke K: Recognition and treatment of the proximal thoracic curve in adolescent idiopathic scoliosis treated with Cotrel-Dubousset instrumentation. Spine (Phila Pa 1976) 19:1589-1597, 1994

9. Luhmann SJ, Sucato DJ, Johnston CE, Richards BS, Karol LA: Radiographic assessment of shoulder position in 619 idiopathic scoliosis patients: can T1 tilt be used as an intraoperative proxy to determine postoperative shoulder balance? J Pediatr Orthop [epub ahead of print], 2015

10. Matsumoto M, Watanabe K, Hosogane N, Kawakami N, Tsuji T, Uno K, et al: Postoperative distal adding-on and related factors in Lenke type 1A curve. Spine (Phila Pa 1976) 38:737-744, 2013
11. Matsumoto M, Watanabe K, Kawakami N, Tsuji T, Uno K, Suzuki T, et al: Postoperative shoulder imbalance in Lenke Type 1A adolescent idiopathic scoliosis and related factors. BMC Musculoskelet Disord 15:366, 2014

12. Menon KV, Tahasildar N, Pillay HM, Anbuselvam M, Jayachandran RK: Patterns of shoulder imbalance in adolescent idiopathic scoliosis: a retrospective observational study. J Spinal Disord Tech 27:401-408, 2014

13. Namikawa T, Matsumura A, Kato M, Hayashi K, Nakamura $\mathrm{H}$ : Radiological assessment of shoulder balance following posterior spinal fusion for thoracic adolescent idiopathic scoliosis. Scoliosis 10 (Suppl 2):S18, 2015

14. Ono T, Bastrom TP, Newton PO: Defining 2 components of shoulder imbalance: clavicle tilt and trapezial prominence. Spine (Phila Pa 1976) 37:E1511-E1516, 2012

15. Qiu Y, Liu Z, Zhu F, Wang B, Yu Y, Zhu Z, et al: Comparison of effectiveness of halo-femoral traction after anterior spinal release in severe idiopathic and congenital scoliosis: a retrospective study. J Orthop Surg 2:23, 2007

16. Risser JC: The iliac apophysis; an invaluable sign in the management of scoliosis. Clin Orthop 11:111-119, 1958

17. Suk SI, Kim WJ, Lee CS, Lee SM, Kim JH, Chung ER, et al: Indications of proximal thoracic curve fusion in thoracic adolescent idiopathic scoliosis: recognition and treatment of double thoracic curve pattern in adolescent idiopathic scoliosis treated with segmental instrumentation. Spine (Phila Pa 1976) 25:2342-2349, 2000

18. Wang Y, Hansen ES, Høy K, Wu C, Bünger CE: Distal adding-on phenomenon in Lenke 1A scoliosis: risk factor identification and treatment strategy comparison. Spine (Phila Pa 1976) 36:1113-1122, 2011

19. Yagi M, Takemitsu M, Machida M: Clavicle chest cage angle difference (CCAD): a novel predictor of postoperative shoulder imbalance in patients with adolescent idiopathic scoliosis. Spine (Phila Pa 1976) 38:E705-E712, 2013

20. Yang S, Feuchtbaum E, Werner BC, Cho W, Reddi V, Arlet V: Does anterior shoulder balance in adolescent idiopathic scoliosis correlate with posterior shoulder balance clinically and radiographically? Eur Spine J 21:1978-1983, 2012

\section{Disclosure}

The authors report no conflict of interest concerning the materials or methods used in this study or the findings specified in this paper.

\section{Author Contributions}

Conception and design: Cho, CS Lee. Acquisition of data: Lim. Analysis and interpretation of data: Cho, Hwang, Lim. Drafting the article: Cho. Critically revising the article: Cho. Reviewed submitted version of manuscript: CS Lee, Lim, DH Lee. Approved the final version of the manuscript on behalf of all authors: Cho. Statistical analysis: Hwang, Lim. Administrative/ technical/material support: CS Lee, Hwang, DH Lee. Study supervision: CS Lee, DH Lee.

\section{Correspondence}

Jae Hwan Cho, Department of Orthopedic Surgery, Asan Medical Center, University of Ulsan College of Medicine, 388-1 PungNap-2-dong, SongPa-gu, Seoul, South Korea. email: doctork78@hanmail.net. 